PROCEEDINGS OF THE

AMERICAN MATHEMATICAL SOCIETY

Volume 134, Number 7 , Pages 1881-1889

S 0002-9939(05)08261-4

Article electronically published on December 19, 2005

\title{
EVALUATIONS OF A CLASS OF DOUBLE $L$-VALUES
}

\author{
DAVID TERHUNE
}

(Communicated by Wen-Ching Winnie Li)

\begin{abstract}
An analytic proof of an evaluation theorem for the "convolution"type double $L$-values of non-principal characters is given. Along the way, Dirichlet character analogues of generalized single and double polylogarithms are defined. The monodromies of these functions play a pivotal role.
\end{abstract}

\section{INTRODUCTION}

In this paper, we employ the symbol $\sum_{m, n=1}^{\infty}$ to denote

$$
\lim _{M \rightarrow \infty} \lim _{N \rightarrow \infty} \sum_{m=1}^{M} \sum_{n=1}^{N}
$$

We will use the standard notation $e(x)=e^{2 \pi i x}$. We denote by $\varphi(n)$ the value of the Euler totient function at $n \in \mathbb{Z}_{+}$.

For any Dirichlet characters $\chi, \psi$, and $a, b \in \mathbb{Z}_{+}$, we define single and double $L$-values (also called multiple $L$-values of depth 1 and 2)

$$
L\left(\begin{array}{l}
\chi \\
a
\end{array}\right)=\sum_{n=1}^{\infty} \frac{\chi(n)}{n^{a}}, \quad L\left(\begin{array}{c}
\chi, \psi \\
a, b
\end{array}\right)=\sum_{m, n=1}^{\infty} \frac{\chi(m) \psi(n)}{m^{a}(m+n)^{b}},
$$

respectively. It has been shown previously that the last sum converges if $\psi \neq \chi_{0}$, and converges absolutely if $b>1$ [1, 8]. It turns out that rearrangement of the limit symbols leaves the sum invariant. In this paper, the term double $L$-value will refer to a sum of the second type.

Multiple $L$-values have previously been defined more generally [1, and have been shown to have an extensive and interesting algebraic structure [4, 7. In the special case $\chi=\psi=1, L(\chi, \psi ; a, b)$ equals the double zeta value $\zeta(a, b)$. The connections of the multiple zeta values with motives, knot theory, and quantum physics [3, 11] provide some of the motivation for this area of research.

Our main result was essentially proven in [ $[$ ] using more elementary methods; we give here a proof using complex function theory. In the last section, an explicit formula for a special case of the evaluations promised by the theorem is given. This formula was calculated using the method of our proof, and holds for quite general Dirichlet characters.

Received by the editors August 27, 2004 and, in revised form, February 9, 2005.

2000 Mathematics Subject Classification. Primary 11M41; Secondary 11B68, 11B75, 11Y35, $33 \mathrm{E} 20$.

Key words and phrases. Multiple $L$-values, multiple zeta values, generalized multiple polylogarithm. 


\section{MAIN RESULTS}

It is well-known that for any Dirichlet character $\chi$ and $a \in \mathbb{Z}_{+}$such that $\chi(-1)=$ $(-1)^{a}$, then

$$
L\left(\begin{array}{l}
\chi \\
a
\end{array}\right)=A \pi^{a},
$$

for some $A \in \mathbb{Q}(e(1 / D))$, where $D=\operatorname{cond}(\chi)$. Our main result is the following extension of this fact.

Theorem 1. Let $\chi$ (respectively $\psi$ ) be a non-principal Dirichlet character of conductor $D$ (respectively $E$ ), and $a, b \in \mathbb{Z}_{+}$. Set $w=a+b, F=\operatorname{lcm}\{D, E\}$, $m=\operatorname{lcm}\{D, E, \varphi(D), \varphi(E)\}$, and $K=\mathbb{Q}(i, e(1 / m))$. If

$$
\chi \psi(-1)=(-1)^{w-1},
$$

then $L(\chi, \psi ; a, b)$ is a $K$-linear finite combination of products of positive integer values of $L$-series of characters with conductors dividing $F$.

Proof. Let $\omega$ be a non-principal Dirichlet character with conductor $D$, and let $\mathbb{H}=\{z \in \mathbb{C}: \Im(z)>0\}$ be the upper half-plane. For $\tau \in \mathbb{H}$, define

$$
H\left(\begin{array}{c}
\omega \\
0
\end{array}\right)(\tau)=\sum_{n=1}^{\infty} \omega(n) e(n \tau) .
$$

By expanding in residue classes, and using geometric series, this is seen to equal

$$
\frac{1}{1-e(D \tau)} \sum_{k=1}^{D} \omega(k) e(k \tau),
$$

providing a meromorphic continuation to the complex plane, and showing

$$
H\left(\begin{array}{c}
\omega \\
0
\end{array}\right)(-\tau)=-\omega(-1) H\left(\begin{array}{l}
\omega \\
0
\end{array}\right)(\tau) .
$$

In addition,

$$
\lim _{\tau \rightarrow \pm 1} H\left(\begin{array}{c}
\omega \\
0
\end{array}\right)(\tau)=-\frac{1}{D} \sum_{k=1}^{D} k \omega(k) .
$$

Hence, $H(\omega ; 0)$ is, in fact, analytic at \pm 1 , with value lying in $\mathbb{Q}(\omega)$.

Suppose $f$ is analytic on a simply connected domain $\Delta \ni 0$. For $\tau \in \Delta$, we define the operator

$$
I(f)(\tau)=2 \pi i \int_{[0, \tau]} f(w) d w,
$$

where the path lies in $\Delta$. Note that if $f(\tau)$ satisfies

$$
f(-\tau)=(-1)^{m} f(\tau),
$$

whenever $\tau,-\tau \in \Delta$, then it follows that

$$
I(f)(-\tau)=(-1)^{m+1} I(f)(\tau),
$$

for these $\tau$.

Let $\epsilon>0$. Let $\Delta$ be a simply connected domain containing $\mathbb{H}$ and a smooth path $\gamma$ starting at 0 and ending at $-1-i \epsilon$ such that $\operatorname{tr}(\gamma)-\{0\}$ lies in $\{z \in \mathbb{C}: \Im(z)<0\}$. 
For Dirichlet characters $\chi, \psi$, and $c, d \in \mathbb{Z}_{+}$, define

$$
H\left(\begin{array}{l}
\chi \\
c
\end{array}\right)(\tau)=I^{c} H\left(\begin{array}{l}
\chi \\
0
\end{array}\right)(\tau)
$$

and

$$
H\left(\begin{array}{c}
\chi, \psi \\
c, d
\end{array}\right)(\tau)=I^{d}\left(H\left(\begin{array}{l}
\psi \\
0
\end{array}\right) H\left(\begin{array}{l}
\chi \\
c
\end{array}\right)\right)(\tau) .
$$

For $b^{\prime} \in \mathbb{Z}_{+}$(with value to be chosen later), set

$$
J(\tau)=H\left(\begin{array}{c}
\chi, \psi \\
a, b^{\prime}
\end{array}\right)(\tau) .
$$

Lemma 1. For $\tau \in \mathbb{H}, J(\tau)$ equals

$$
\sum_{m, n=1}^{\infty} \frac{\chi(m) \psi(n)}{m^{a}(m+n)^{b^{\prime}}} e((m+n) \tau)-\sum_{k=0}^{b^{\prime}-1} \frac{(2 \pi i \tau)^{k}}{k !} L\left(\begin{array}{c}
\chi, \psi \\
a, b^{\prime}-k
\end{array}\right),
$$

plus a $\mathbb{Q}$-linear finite combination of products of functions in the set

$$
\left\{(2 \pi i \tau)^{c}, H\left(\begin{array}{l}
\omega \\
c
\end{array}\right)(\tau), L\left(\begin{array}{c}
\omega \\
c
\end{array}\right): c \in \mathbb{Z}_{+}, \omega \in\{\chi, \psi\}\right\} .
$$

Proof. Using uniform convergence, one sees by induction

$$
H\left(\begin{array}{l}
\chi \\
a
\end{array}\right)(\tau)=\sum_{n=1}^{\infty} \frac{\chi(n)}{n^{a}} e(n \tau)-\sum_{k=0}^{a-1} \frac{(2 \pi i \tau)^{k}}{k !} L(\chi, a-k) .
$$

By multiplying by $H(\psi ; 0)(\tau)$, applying $I b^{\prime}$ times, and using the calculus identity

$$
\int x^{k} e^{n x} d x=(-1)^{k} e^{n x} \sum_{l=0}^{k} \frac{k !}{l !} \frac{(-x)^{l}}{n^{k-l+1}}
$$

(after making the substitution $x=2 \pi i \tau$ ), one obtains the result.

Note that by uniform Convergence And Lemma 1,

$$
\lim _{\tau \rightarrow 1} H\left(\begin{array}{c}
\chi, \psi \\
a, b
\end{array}\right)(\tau)
$$

is a $\mathbb{Q}$-linear combination of products of single and double $L$-values of the characters $\chi, \psi$. By induction, one also sees easily

Lemma 2. The function $J(\tau)$ continues analytically to $\Delta$.

We now require that the simply connected domain $\Delta$ contain no poles of either $H(\chi ; 0)$ or $H(\psi ; 0)$. Define

$$
J(1)=\lim _{\substack{\tau \rightarrow 1 \\ \tau \in \mathbb{H}}} J(\tau),
$$

$J(-1-i \epsilon)$ to be the value of the analytic continuation of $J$ along $\gamma$, and

$$
J(-1)=\lim _{\epsilon \rightarrow 0} J(-1-i \epsilon) .
$$

For $c_{1}, \ldots, c_{d} \in \mathbb{Z}$, we shall denote the generalized multiple polylogarithm of depth $d$ by

$$
\operatorname{Li}_{c_{1}, \ldots, c_{d}}(z)=\sum_{n_{1}, \ldots, n_{d}=1}^{\infty} \prod_{j=1}^{d} z^{n_{j}}\left(\sum_{k=1}^{j} n_{k}\right)^{-c_{j}}
$$


As in [6], we view Li as a solution of the formal KZ-equation

$$
\frac{d F}{d z}=\left(\frac{X}{z}+\frac{Y}{1-z}\right) F
$$

where $F$ lies in the non-commutative ring of formal power series $\mathcal{A}[X, Y]$, and $\mathcal{A}$ is the set of functions analytic on the unit disk.

It was shown by $\mathrm{R}$. Hain [5] that, for $c \in \mathbb{Z}_{+}$, the monodromy of $\operatorname{Li}_{c}(z)$ about $z=1$ is the function

$$
2 \pi i \frac{\log ^{c-1}(z)}{(c-1) !} .
$$

Hain's method may be extended, using (5), to the generalized multiple polylogarithms. These monodromies may also be computed using the coefficients of the connection matrices treated by J. Okuda in [6].

We will generalize Li to non-principal Dirichlet characters for depth $\leq 2$; our generalization also solves an analogous formal KZ-equation. For $c \in \mathbb{Z}_{+}$, define a polylogarithm associated to a character

$$
\operatorname{Li}\left(\begin{array}{l}
\chi \\
c
\end{array}\right)(z)=\sum_{n=1}^{\infty} \frac{\chi(n)}{n^{c}} z^{n}
$$

By making use of the finite Fourier expansion of a Dirichlet character $\chi$,

$$
\chi(n)=\sum_{k=1}^{D} \theta_{k}(\chi) e(k n / D), \quad \theta_{k}(\chi) \in \mathbb{Q}(e(1 / D))
$$

(where $D$ is the conductor of $\chi$ ), it follows that the monodromy of this function about $e(k / D)$ is $2 \pi i$ times a $K$-linear finite combination of products of functions in the set $\left\{2 \pi i, \log ^{m}(z): m \in \mathbb{Z}_{+}\right\}$.

We define a generalized double polylogarithm associated to Dirichlet characters

$$
\operatorname{Li}\left(\begin{array}{c}
\chi, \psi \\
c, d
\end{array}\right)(z)=\sum_{m, n=1}^{\infty} \frac{\chi(m) \psi(n)}{m^{c}(m+n)^{d}} z^{m+n}
$$

for $c, d \in \mathbb{Z}_{+}$. Note that our functions $H(\tau)=\operatorname{Li}(e(\tau))$. Under this substitution, our path in $\Delta$ is mapped to a loop based at 1 . This connects the value $H(-1)$ with the monodromy of $\mathrm{Li}$. We require the following.

Lemma 3. The sum of the monodromies of $\operatorname{Li}(\chi, \psi ; c, d)(z)$ about all roots of unity is a K-linear finite combination of products of functions in the set

$$
\left\{2 \pi i, \log (z), L\left(\begin{array}{c}
\omega \\
a
\end{array}\right), \operatorname{Li}\left(\begin{array}{c}
\omega \\
a
\end{array}\right)(z): \operatorname{cond}(\omega) \mid G, a \in \mathbb{Z}_{+}\right\}
$$

where $G=[D, E]$, and $\log$ denotes the branch of the logarithm analytic on $\{z \in$ $\mathbb{C}:|z|=1, z \neq 1\}$, and such that $\log (1)=0$. Furthermore, $2 \pi i$ appears in each summand to at least the first power, and any term not an integral multiple of

$$
2 \pi i L\left(\begin{array}{c}
\chi \psi \\
c+d-1
\end{array}\right)
$$

contains either $\log (z)$ or $(2 \pi i)^{2}$ as a factor. 
Proof. The proof of the first statement is by induction on $d$. In the case $d=1$, we write

$$
\operatorname{Li}\left(\begin{array}{c}
\chi, \psi \\
c, 1
\end{array}\right)(z)=\sum_{k=1}^{D} \sum_{l=1}^{E} \theta_{k}(\chi) \theta_{l}(\psi) \int_{0}^{z} \operatorname{Li}_{c}(e(k / D) w) \frac{e(l / E)}{1-e(l / E) w} d w
$$

where $D$ (respectively $E$ ) denotes the conductor of $\chi$ (respectively $\psi$ ). The terms with $c=1, k / D=l / E$ each equal an element of $K$ times $\operatorname{Li}_{1,1}(e(k / D) z)$, and the monodromy of this function is easily calculated via the identity

$$
\operatorname{Li}_{1,1}(z)=\frac{1}{2} \operatorname{Li}_{1}(z)^{2}
$$

(proven in [2]), and takes the required form. For the other terms, we follow Hain. Let $\varepsilon>0$ be small, and $\sigma$ a circular clockwise path beginning and ending at $e(j / F)(1-\varepsilon)$ of radius $\varepsilon$. We consider the integral

$$
\int_{0}^{z}+\int_{z}^{e(j / F)(1-\varepsilon)}+\int_{\sigma}+\int_{e(j / F)(1-\varepsilon)}^{z} \operatorname{Li}_{c}(e(k / D) w) \frac{e(l / E)}{1-e(l / E) w} d w .
$$

The first piece is simply the original function. If $k / D \neq l / E$, we compute the third piece by evaluating a contour integral. In the case $c>1$ and $k / D=l / E$, we parametrize to find the third piece approaches zero as $\varepsilon \rightarrow 0$. The last piece minus the second piece is

$$
\int_{e(j / F+k / D)(1-\varepsilon)}^{e(k / D) z} g(w) \frac{e(l / E-k / D)}{1-e(l / E-k / D) w} d w,
$$

where $g$ is the monodromy of $\operatorname{Li}_{c}$ about $e(j / F+k / D$ ) (which of course vanishes if $j / F+k / D \notin \mathbb{Z}$ ). By expanding in geometric series and applying (4), we write this in terms of the required functions and values of classical polylogarithms evaluated at roots of unity, which by Proposition 3 of [8], can be written in terms of $L$-series values with conductors having the prescribed bound. This produces the required form.

In the case $d>1$, we write

$$
\operatorname{Li}\left(\begin{array}{c}
\chi, \psi \\
c, d
\end{array}\right)(z)=\int_{0}^{z} \operatorname{Li}\left(\begin{array}{c}
\chi, \psi \\
c, d-1
\end{array}\right)(w) \frac{d w}{w} .
$$

By an analogous procedure, we reduce to integrating $1 / w$ times the monodromy of $\operatorname{Li}(\chi, \psi ; c, d-1)$ about roots of unity, which by the induction hypothesis, and (4), takes the required form. The other assertions are easy consequences of this procedure.

The condition (2) implies that

$$
J(-\tau)=-J(\tau)
$$

for $\tau \in \Delta$, with the values correctly defined (this plays the role of equation (2.11) of [10]). By letting $\varepsilon \rightarrow 0$, this holds for $\tau=1$ also, given our definitions.

Let $h(z)$ be the sum of the monodromies of $\operatorname{Li}\left(\chi, \psi ; a, b^{\prime}\right)(z)$ about all roots of unity. Writing

$$
J(\tau)=I H\left(\begin{array}{c}
\chi, \psi \\
a, b^{\prime}-1
\end{array}\right),
$$

and making the change of variables $z=e(\tau)$, we see $J(-1)$ equals $J(1)+h(1)$. By Lemma 3, (10) and (2), $h(1)$ is $(2 \pi i)^{2}$ times a $K$-linear finite combination of 
products of powers of $2 \pi i$ and positive-integer values of $L$-series of characters with conductors dividing $F$.

For $\tau \in \mathbb{H}$, by Lemma 1 we can write

$$
\begin{aligned}
J(\tau)=\operatorname{Li}\left(\begin{array}{c}
\chi, \psi \\
a, b^{\prime}
\end{array}\right)(e(\tau))-\sum_{k=0}^{b^{\prime}-1} L\left(\begin{array}{c}
\chi, \psi \\
a, b^{\prime}-k
\end{array}\right) \frac{(2 \pi i \tau)^{k}}{k !} \\
\quad+\sum_{\mu \in I}(2 \pi i \tau)^{\mu} \sum_{\omega=\chi, \psi}\left\{g_{\mu}+h_{\mu} \operatorname{Li}\left(\begin{array}{c}
\omega \\
c_{\mu, \omega}
\end{array}\right)(e(\tau))\right\},
\end{aligned}
$$

where $I \subset \mathbb{Z}_{\geq 0}$ is a finite set, each $c_{\mu, \omega} \in \mathbb{Z}_{+}$, and each $g_{\mu}, h_{\mu}$ is a $\mathbb{Q}$ - linear finite combination of positive integer values of $L$-series of the characters $\chi, \psi$. Note that each summand in this expression continues analytically to $\Delta$.

We make use of the elementary fact that for $k \in \mathbb{Z}, c \in \mathbb{R}$,

$$
(2 \pi i \tau)^{k} e(c \tau)+(-2 \pi i \tau)^{k} e(-c \tau)= \begin{cases}2(2 \pi i \tau)^{k} \cos (2 \pi c \tau), & k \text { even } \\ 2 i(2 \pi i \tau)^{k} \sin (2 \pi c \tau), & k \text { odd }\end{cases}
$$

By this, (7), (8), and Lemma 3, we have for $\tau \in \mathbb{C}$ such that $\tau,-\tau \in \Delta$,

$$
\begin{aligned}
0=J(\tau)+J(-\tau)= & 2 \operatorname{Li}\left(\begin{array}{c}
\chi, \psi \\
a, b^{\prime}
\end{array}\right)(e(\tau))-2 \sum_{\substack{k=0 \\
2 \mid k}}^{b^{\prime}-1} \frac{(2 \pi i \tau)^{k}}{k !} L\left(\begin{array}{c}
\chi, \psi \\
a, b^{\prime}-k
\end{array}\right) \\
& +\sum_{\substack{\mu \in I \\
2 \mid \mu}} 2(2 \pi i \tau)^{\mu} \sum_{\omega=\chi, \psi}\left\{g_{\mu}+h_{\mu} \sum_{n=1}^{\infty} \frac{\omega(n)}{n^{c_{\mu, \omega}}} \cos (2 \pi n \tau)\right\} \\
& +\sum_{\substack{\mu \in I \\
2 \nmid \mu}} 2 i(2 \pi i \tau)^{\mu} \sum_{\omega=\chi, \psi}\left\{g_{\mu}+h_{\mu} \sum_{n=1}^{\infty} \frac{\omega(n)}{n^{c_{\mu, \omega}}} \sin (2 \pi n \tau)\right\} .
\end{aligned}
$$

Since this also holds for $\tau=1$, with our definitions, we have by uniform convergence

$$
\sum_{\substack{k=2 \\
2 \mid k}}^{b^{\prime}-1} \frac{(2 \pi i)^{k}}{k !} L\left(\begin{array}{c}
\chi, \psi \\
a, b^{\prime}-k
\end{array}\right)=\frac{1}{2} h(1)+\sum_{\substack{\mu \in I \\
2 \mid \mu}}(2 \pi i)^{\mu} \sum_{\omega=\chi, \psi}\left\{g_{\mu}+h_{\mu} L\left(\begin{array}{c}
\omega \\
c_{\mu, \omega}
\end{array}\right)\right\} .
$$

Setting $\tau=0$ in (9) shows the $\mu=0$ term here vanishes. Now, since $(2 \pi i)^{2}$ is a factor of $h(1)$, we can divide by $(2 \pi i)^{2}$ to obtain

$$
\begin{aligned}
\sum_{\substack{k=2 \\
2 \mid k}}^{b^{\prime}-1} \frac{(2 \pi i)^{k-2}}{k !} L\left(\begin{array}{c}
\chi, \psi \\
a, b^{\prime}-k
\end{array}\right)=\frac{1}{2} & \frac{h(1)}{(2 \pi i)^{2}} \\
& +\sum_{\substack{\mu>0 \\
2 \mid \mu}}(2 \pi i)^{\mu-2} \sum_{\omega=\chi, \psi}\left\{g_{\mu}+h_{\mu} L\left(\begin{array}{c}
\omega \\
c_{\mu, \omega}
\end{array}\right)\right\} .
\end{aligned}
$$

We thus have linear combinations of our double $L$-values written in terms of singleindex sums. We require the following sequence inversion (which is analogous to Lemma 7 of 9$]$ ). 
Lemma 4. Suppose $\left\{a_{n}\right\}_{n=1}^{\infty},\left\{b_{n}\right\}_{n=1}^{\infty}$ are sequences of complex numbers satisfying

$$
\sum_{m=0}^{n-1} a_{n-m} \frac{(2 \pi i)^{2 m}}{(2 m+2) !}=b_{n}
$$

for each $n \in \mathbb{Z}_{+}$. For $m \in \mathbb{Z}_{\geq 0}$, define rational numbers $C_{0}=2$,

$$
C_{2 m}=\frac{-B_{2 m}}{m(2 m-2) !},
$$

for $m \geq 1$, where $B_{j}$ denotes the $j$ th Bernoulli number. Then

$$
a_{n}=\sum_{m=0}^{n-1} C_{2 m}(2 \pi i)^{2 m} b_{n-m}
$$

for each $n \in \mathbb{Z}_{+}$.

Proof. Set $f(x):=e^{x}\left(x /\left(e^{x}-1\right)\right)^{2}$. Using geometric series one calculates

$$
\int_{0}^{\infty} x^{s-3} f(x) d x=\Gamma(s) \zeta(s-1)
$$

for $\Re(s)>2$, providing a meromorphic continuation of $\zeta$. Now, truncating the integral and calculating residues, using the fact that $\zeta(-n)=-B_{n+1} /(n+1)$, shows that the coefficient of $x^{k}$ in the Maclaurin series expansion of $f(x)$ is one if $k=0$, zero if $k=1$, and

$$
\frac{(-1)^{k-1} B_{k}}{k(k-2) !}
$$

for $k \geq 2$.

Next, using generating functions, one sees from a power series expansion that

$$
\begin{aligned}
(2 \pi i x)^{-2}(\cosh (2 \pi i x)-1) \sum_{n=1}^{\infty} a_{n} x^{2 n} & =\sum_{\substack{m=0 \\
n=1}}^{\infty} \frac{(2 \pi i)^{2 m}}{(2 m+2) !} a_{n} x^{2 m+2 n} \\
& =\sum_{n=1}^{\infty} \sum_{m=0}^{n-1} \frac{(2 \pi i)^{2 m}}{(2 m+2) !} a_{n-m} x^{2 n}=\sum_{n=1}^{\infty} b_{n} x^{2 n} .
\end{aligned}
$$

Note that

$$
\frac{(2 \pi i x)^{2}}{\cosh (2 \pi i x)-1}=2 e^{2 \pi i x}\left(\frac{2 \pi i x}{e^{2 \pi i x}-1}\right)^{2}=2 f(2 \pi i x)
$$

Hence,

$$
\sum_{n=1}^{\infty} a_{n} x^{2 n}=2 f(2 \pi i x) \sum_{n=1}^{\infty} b_{n} x^{2 n}
$$

whence the conclusion follows by multiplying power series and equating the coefficients, using (11).

Set $g=\left(1+(-1)^{b+1}\right) /(-2)$. We Apply Lemma 4 in the case

$$
a_{n}=L\left(\begin{array}{c}
\chi, \psi \\
a, g+2 n
\end{array}\right)
$$

with $b_{n}$ given by the RHS of (10), after setting $b^{\prime}=g+2(n+1)$. This proves Theorem 1. 


\section{SOME EXAMPLES}

The following examples were computed using the method of our proof. All characters are assumed primitive and non-trivial. If $\chi$ has conductor $D$, then

$$
\begin{aligned}
& L\left(\begin{array}{l}
\chi \\
1
\end{array}\right)=-\frac{\pi i}{D^{2}} \sum_{k=1}^{D} k^{2} \theta_{k}(\chi), \quad \chi(-1)=-1, \\
& L\left(\begin{array}{l}
\chi \\
2
\end{array}\right)=\frac{2}{3} \frac{\pi^{2}}{D^{3}} \sum_{k=1}^{D} k^{3} \theta_{k}(\chi), \quad \chi(-1)=1 .
\end{aligned}
$$

Now suppose, in addition, $\psi$ has conductor $E, \chi(-1)=1, \psi(-1)=-1$, and $(D, E)=1$. With a couple of applications of (12), our technique yields

$$
\begin{aligned}
L\left(\begin{array}{c}
\chi, \psi \\
1,1
\end{array}\right) & =-\frac{1}{2 \pi i} L\left(\begin{array}{c}
\chi \psi \\
3
\end{array}\right)-\frac{\pi i}{D^{2}} \sum_{k=1}^{D} k^{2} \theta_{k}(\chi) \operatorname{Li}\left(\begin{array}{c}
\psi \\
1
\end{array}\right)(e(k / D)) \\
& +\frac{1}{D} \sum_{k=1}^{D} k \theta_{k}(\chi) \operatorname{Li}\left(\begin{array}{c}
\psi \\
2
\end{array}\right)(e(k / D))-\frac{\pi i}{E^{2}} \sum_{k=1}^{E} k^{2} \theta_{k}(\psi) \operatorname{Li}\left(\begin{array}{c}
\chi \\
1
\end{array}\right)(e(-k / E)) .
\end{aligned}
$$

The first term on the RHS seems interesting. It should be possible with our methods to derive such formulas at any positive integer point, in the situation (2) (although we have not proven this).

Remark 1. The techniques herein should, in essence, apply to higher-depth multiple $L$-values, and to principal characters. The author is continuing his investigation into this matter.

\section{ACKNOWLEDGEMENTS}

The author is indebted to a paper of Hirofumi Tsumura [10, which served largely as the inspiration for these techniques. In addition, he is grateful for a conversation with Jianqiang Zhao concerning analytic continuations of multiple polylogarithms (who has written a paper on the subject [12]). The author would also like to thank the referee for some very helpful suggestions.

\section{REFERENCES}

[1] T. Arakawa, M. Kaneko, On Multiple L-values J. Math. Soc. Japan. 56 (2004), no. 4, 967-991. MR.2091412

[2] J. Borwein, D. Broadhurst, J. Kamnitzer, Central Binomial Sums, Multiple Clausen Values, and Zeta Values, Experimental Math., 10 (2001) 1, 25-34. MR1821569 (2002k:11105)

[3] J. Borwein, R. Girgensohn, Evaluation of Triple Euler Sums, Electron. J. Combin. 3 (1996) 1, Research Paper 23, 27 pp. MR 1401442 (97d:11137)

[4] A. B. Goncharov, Multiple Polylogarithms, Cyclotomy, and Modular Complexes, Math. Res. Lett., 5 (1998) 4, 497-516. MR.1653320(2000c:11108)

[5] R. Hain, Classical Polylogarithms, Proc. Symp. Pure Math. 55 (1994), 3-42. MR.1265550 (94k:19002)

[6] J. Okuda, Duality Formulas of the Special Values of Multiple Polylogarithms Bull. London Math. Soc. 37 (2005), no. 2, 230-242. MR2119023

[7] G. Racinet, Doubles Mélanges des Polylogarithmes Multiples aux Racines de L'unité (French) [Double Shuffles of Multiple Polylogarithms at Roots of Unity], Publ. Math. Inst. Hautes Études, 95 (2002), 185-231. MR.1953193 (2004c:11117)

[8] D. Terhune, Evaluations of Double L-values, J. Num. Th. 105 (2004), 275-301. MR2040159 (2005f:11200) 
[9] H. Tsumura, Combinatorial Relations for Euler-Zagier Sums, Acta Arith. 111 (2004), 27-42. MR2038060 (2005a:11140)

[10] H. Tsumura, On Mordell-Tornheim Zeta Values, Proc. Amer. Math. Soc. 133 (2005), no. 8, 2387-2393. MR 2138881

[11] D. Zagier, Values of Zeta Functions and their Applications, in: A. Joseph, et al. (Eds.), Proceedings of the First European Congress of Mathematics, Paris, Vol. II (1994), Birkhäuser Verlag, 497-512. MR1341859 (96k:11110)

[12] J. Zhao, Analytic Continuation of Multiple Polylogarithms, preprint arXiv:math.AG/ 0302054 .

Department of Mathematics, The Pennsylvania State University, 109 McAllister Building, University Park, Pennsylvania 16802 